\title{
Reference equation for the incremental shuttle walk test in Japanese adults
}

Masatoshi Itaki, PT, MS ${ }^{\mathrm{a}, \mathrm{b}}$, Ryo Kozu, PT, PhD ${ }^{\mathrm{b}}$, Kenichiro Tanaka, PT ${ }^{\mathrm{b}, \mathrm{c}}$, Hideaki, Senjyu, PT,

$\mathrm{PhD}^{\mathrm{d}}$, the Clinical Pulmonary Functions Committee of the Japanese Respiratory Society, the

Development Committee for Reference Values for the Field Walking Tests of the Japanese Society

for Respiratory Care and Rehabilitation

${ }^{a}$ Department of Rehabilitation Medicine, Tagami Hospital, 2-14-15, Tagami, Nagasaki, Nagasaki,

851-0251, Japan.

${ }^{\text {b}}$ Department of Cardiopulmonary Rehabilitation Science, Nagasaki University Graduate School of

Biomedical Sciences, 1-7-1, Sakamoto, Nagasaki, Nagasaki, 852-8520, Japan.

'Faculty of Welfare and Health Sciences, Oita University, 700, Dannobaru, Oita, Oita, 870-1192, Japan.

${ }^{\mathrm{d} D e p a r t m e n t}$ of Respiratory Care and Rehabilitation Center, Fukujuji Hospital, 3-1-24, Matsuyama, Kiyose, Tokyo.

\section{Corresponding author: Ryo Kozu}

Department of Cardiopulmonary Rehabilitation Science, Nagasaki University Graduate School of 
Biomedical Sciences, 1-7-1, Sakamoto, Nagasaki, Nagasaki, 852-8520, Japan

e-mail: ryokozu@nagasaki-u.ac.jp

Tel and Fax: +81-95-819-7967 


\section{ABSTRACT}

Background: The incremental shuttle walk test (ISWT) is widely used in clinical and research settings. However, there are no reference equations to predict the walk distance achieved in the ISWT (ISWD) for healthy Japanese adults. We aimed to establish a reference equation for the ISWD prediction in Japanese adults.

Methods: The sample comprised 590 healthy Japanese subjects (237 male). All subjects performed the ISWT twice, and their anthropometric and demographic data were collected, including gender, age, height, weight, and body mass index (BMI).

Results: Subjects walked 640 [490-793] $\mathrm{m}$ in the ISWT. The ISWD correlated $(\mathrm{p}<0.001$ for all) with age $(r=-0.51)$, gender $(r=0.56)$, weight $(r=0.39)$, and height $(r=0.62)$, but not with BMI $(r$ $=-0.01, \mathrm{p}=0.74)$. The stepwise multiple regression model showed that age, gender, and height were independent contributors to the ISWT in healthy subjects, explaining $50 \%$ of the variability. The reference equation for the ISWD was: $\operatorname{ISWD}(\mathrm{m})=-4.894-4.107 \times$ Age (years $)+131.115 \times$ Gender $+4.895 \times$ Height $(\mathrm{cm})$, where male gender $=1$.

Conclusion: We have established a reference equation for the ISWD prediction in Japanese adults. The prediction accuracy was high $\left(\mathrm{R}^{2}=50 \%\right)$, and a reference equation was established using anthropometric and demographic variables that can be easily assessed in clinical settings. The reference equation developed in this study will be useful for evaluating the magnitude of exercise intolerance in Japanese adults. 
Keywords: Reference value, Incremental shuttle walk test, Exercise test, Statistical regression 
Abbreviations: 6MWT (six- minute walk test), ISWT (incremental shuttle walk test), $\dot{\mathrm{VO}}_{2}$ peak (peak oxygen uptake), $\mathrm{SpO}_{2}$ (percutaneous oxygen saturation), $\mathrm{PR}$ (pulse rate), \%HRmax (percentage of the predicted HRmax), ISWD (the walk distance in ISWT), COPD (chronic obstructive pulmonary disease), BMI (body mass index), 15mISWRT (15m incremental shuttle walk and run test) 


\section{Introduction}

Exercise intolerance is a common presentation and prognostic indicator in chronic disease.

Therefore a need exists to quantify exercise tolerance, and assess response to therapeutic

interventions. Currently, field exercise tests such as the six-minute walk test (6MWT) and

incremental shuttle walk test (ISWT) are widely used to assess exercise tolerance and prescribe

training intensity in pulmonary rehabilitation programs [1]. Although these tests were developed in

patients with cardiopulmonary diseases $[2,3]$, their use has been validated in a wide range of chronic

diseases $[4,5]$ in part due to their operational simplicity.

The 6MWT is the most widely used in the field walking tests in Japanese clinical setting,

because it is simple and inexpensive to perform [2]. However, the main disadvantage is that the

patient's exercise tolerance is underestimated, as it allows the patient to set the walking speed. As an alternative to self-paced tests and in an attempt to improve standardization and reproducibility, the ISWT was developed by Singh et al. [3]. The ISWT [3] is an externally paced incremental walking test which evaluates maximal exercise capacity. In individuals with chronic obstructive pulmonary disease (COPD), the distance walked (incremental shuttle walk distance [ISWD]) correlates more strongly with peak oxygen uptake $\left(\mathrm{VO}_{2}\right.$ peak) than the 6MWT distance and has good reliability [6]. Therefore, it is possible to use the predicted $\mathrm{VO}_{2}$ peak, from the ISWD, for exercise prescription. The ISWT has been used to assess the response to interventions [7-10], identify oxygen desaturation 
during exertion [11], and predict mortality [12] and exacerbations [13] in chronic respiratory disease.

In healthy individuals, the ISWT has proven useful for quantifying the benefits of a walking training program [14].

Despite the widespread use of the ISWT, there are no reference equations to predict the ISWD achieved by healthy Japanese adults. Such an equation is necessary to determine the magnitude of exercise intolerance.

Although equations exist for the ISWD prediction in European, American, and Brazilian adults [15-18], compared with these populations, Japanese individuals have a smaller physique, (e.g. height, weight, and body mass index (BMI) [19, 20],). In addition, we hypothesized that existing equations may not be applicable to Japanese samples. In this study, we aimed to establish a reference equation for the ISWD prediction in Japanese adults and to compare measured ISWD with predicted ISWD derived from an existing equation (Probst et al.) [18].

\section{Materials and methods}

\subsection{Subjects}

Five hundred and ninety-nine healthy Japanese male and female volunteers, aged between 20 and 90 years, were recruited which include staff at 13 hospitals (Nagasaki, Kitakyushu, Kagoshima, Ise, Kurashiki, Ogaki, Yamaguchi, Okinawa, Oita, and Tokyo), staff and students at four universities (Nagasaki, Kio, Health Science, and Kumamoto Health Science), and participants in community 
health classes for elderly people (total of 20 facilities). Data were collected between September 2013

and October 2015. The study was approved (approval date; 8/8/2013, number; 13080844 ) by the Ethics Review Committee of Nagasaki University Graduate School of Biomedical Sciences. Written informed consent was obtained from all participants.

Exclusion criteria were any health problem that could interfere with the ability to perform physical exercise: e.g. cardiovascular, respiratory, neuromuscular, or musculoskeletal disease; upper respiratory tract infection within the previous month; and history of stroke, severe dementia and mental disorders, and visual and hearing impairment. However, subjects with well-controlled hypertension and former smokers without tobacco-related diseases were included. If no problems were identified during the health screening check, subjects' exercise capacity and anthropometric and demographic data were evaluated. Gender, age, height, weight, and BMI were collected on the day they performed the ISWT.

\subsection{Incremental shuttle walk test}

The ISWT was performed according to the original protocol [3]. Subjects performed the test twice (separated by a rest period of at least 30 minutes). To allow for the known learning effect [21], the results of the second ISWT were recorded for analysis. Subjects were required to walk along a level 10-m course, marked by 2 cones placed 9-m apart at a speed dictated by signals from an audio 
CD. The walking speed increased each minute (initial walking speed was $0.5 \mathrm{~m} / \mathrm{s}$ and increased by $0.17 \mathrm{~m} / \mathrm{s}$ each minute), for a total of 12 levels. Subjects were not permitted to jog or run during the test. The maximum achievable distance is $1020 \mathrm{~m}$. All ISWTs were supervised by a physiotherapist at the facility who was experienced with the ISWT. Criteria for test termination were failure to maintain the required speed or symptoms. Oxygen saturation $\left(\mathrm{SpO}_{2}\right)$, pulse rate (pulse oximeter, Pulsox-Me300, Minolta, Osaka, Japan), and perceived dyspnea and leg fatigue (modified Borg scale [22]) were assessed prior to and immediately upon test termination.

\subsection{Statistical analysis}

The distribution of the data was assessed using Kolmogorov-Smirnov test and frequency histograms. Data are described as median [interquartile range 25\%-75\%]. The Mann-Whitney test was used to compare the subjects' characteristics and measures obtained from the ISWT between males and females. Spearman's correlation coefficients were calculated to examine the relationship between variables (age, gender, height, weight, and BMI) and the ISWD. Multiple linear regression analysis was used to evaluate the best predictor variables for the ISWD and to generate the regression equation.

The measured ISWD was compared with the predicted ISWD derived in a Brazilian sample [18] (subjects aged 18-83 years) using scatter plots and Wilcoxon rank sum test. No comparisons were 
made with other predicted equations because they are only applicable to individuals aged over 40 years or required variables (e.g. lower limb strength or physical activity) that were not assessed in our study [15-17]. The level of statistical significance was set at 5\%. All analyses were performed using the statistical software program IBM SPSS ver. 21 for Windows (IBM SPSS, Tokyo, Japan).

\section{Results}

The study sample comprised 599 subjects. Data from nine subjects were not included because they terminated the test prematurely due to severe joint pain. The characteristics of the remaining 590 subjects (237 males and 353 females) are summarized in Table 1.

Subjects walked 640 [490-793] $\mathrm{m}$ in the ISWT and reached 81 [71-90]\% of their maximal predicted HR (220 - age in years) [23]. In 63 subjects (56 males, 7 females), there was a ceiling effect for the test. In the remaining 527 subjects, the test was terminated because they were unable to keep up with the speed (482 [82\%]) and symptoms (leg fatigue in 28 subjects; dyspnea in nine subjects and other symptoms in eight subjects). Three subjects reported low back and knee pain that ceased immediately on test termination. No other adverse events were reported.

Significant correlations were observed between the ISWD and age $(r=-0.51)$, gender $(r=0.56)$, weight $(r=0.39)$, and height $(r=0.62)(p<0.001$ for all $)$, but no relationship with BMI $(r=-0.01$, $p=0.74)$ was observed. Stepwise multiple regression showed that age, gender, and height were 
independent contributors to the ISWD, explaining $50 \%$ of the variance. Unstandardized coefficients, partial correlations, and significance are shown in Tables 2 and 3. The reference equation for the ISWD was:

$\operatorname{ISWD}(\mathrm{m})=-4.894-4.107 \times$ Age $($ years $)+131.115 \times$ Gender $+4.895 \times$ Height $(\mathrm{cm})$ $($ where male $=1)$

Comparisons between the measured ISWD and ISWD derived in a Brazilian sample are shown in Figure 1. Distances derived using the equation [18] significantly overestimated the measured ISWD. The mean estimated ISWD was 911 [677-1079] m compared to the measured ISWD of 640 $[490-793] \mathrm{m}(\mathrm{p}<0.001)$.

\section{Discussion}

This is the first study to develop a reference equation to predict the ISWD in a healthy Japanese population. A large part (50\%) of the variance in the ISWD was explained by age, gender, and height. The amount of variance explained $\left(\mathrm{R}^{2}=0.5\right)$ was similar to that previously reported [17]. This reference equation is a useful tool in evaluating the ISWT results obtained from patients. Since the ISWT is an externally paced walk test, the interference such as operator encouragement and motivation is lower. Therefore, this reference equation derived in the present study can be useful in other populations. 
Recently, both British and Brazilian researchers have reported a reference equation for the ISWT

[15-18]. Our sample included a larger number of subjects (590 vs 90 to 242) compared to these studies [15-18]. Further, other studies were restricted to subjects aged $\geq 40$ years [15-17]. Moreover, we established an equation using anthropometric and demographic variables which can be easily applied in clinical settings. Therefore, the reference equation we developed will be useful in determining the presence and extent of exercise intolerance in clinical populations.

In our study, the demographic and anthropometric variables identified as independent predictors of the ISWD were age, gender, and height. Our findings are consistent with published data relating to exercise tolerance and walking ability which have shown a relationship with age [24-30], gender $[25,27,28]$, and height $[25,27,28,31]$. The negative influence of advanced age on the ISWD is explained by a decline in skeletal muscle function and $\dot{\mathrm{VO}}_{2}$ peak that typically occurs parallel with aging $[29,30]$. In our study, males walked further than females, as has been described for the ISWT previously $[15,17,18]$, most likely due to males having higher absolute muscle strength and mass and height [32]. Previous studies for the 6MWT and ISWT observed an association between height and walking distance in healthy adults $[15,17,33,34]$. The strong influence of height is attributable to stride length, a major predictor of walking speed [31].

Weight and BMI were not identified as independent predictors of the ISWD in contrast to earlier studies [15-18]. Very few of the subjects in our study were overweight. Further, our sample had a 
narrower weight range and BMI than subjects in studies that developed a prediction equation for the ISWD in European and American samples [15-18]. In studies that generated a reference equation for the 6MWT, Poh et al. [34] reported that equations derived from Caucasian subjects overestimated 6MWD in Singaporeans of Chinese origin due to differences in physique. Regression equations derived in Caucasian subjects [18] overestimated the ISWD for our Japanese sample. Future studies are needed to determine if our equation is applicable to individuals of other Asian origin.

In the present study, subjects reached 81 [71-90]\% of their maximal predicted HR during the ISWT, a value lower than in the previous study [18]. This may be explained by variations in the ISWT protocol. Since the ISWT was developed to assess functional exercise capacity in patients with respiratory diseases, the original protocol consists of 12 levels. However, to avoid the ceiling effect in healthy subjects, Probst et al. [18] used the modified protocol, permitting subjects to run in order to maintain the speed. However, the original ISWT was used consistently with that in clinical settings (i.e. 12 levels, no running) within Japan, and the predominant reason for ISWT termination was the inability to maintain the required speed. In our study, of the 63 subjects $(10.7 \%)$ who completed level 12, most were males aged 20 to 30 years. In previous studies in Japan, Mikawa et al. [35] reported the utility of the $15 \mathrm{~m}$ incremental shuttle walk and run test (15m ISWRT) in young and middle-aged healthy individuals and showed a strong correlation with $\mathrm{VO}_{2}$ peak $(\mathrm{r}=0.86)$ and concluded that the test is valid and safe in evaluating $\mathrm{VO}_{2}$ peak in adults. Our findings suggest that 
the ISWT is not suitable in evaluating exercise tolerance in young healthy males. Therefore,

clinicians and researchers should choose either the ISWT or 15m ISWRT based on the age and expected exercise tolerance of their sample.

Some limitations of our study should be considered. First, consistent with other studies that developed reference values for field walking tests $[15,17,18]$, we used a convenience sample, which is likely to have introduced bias albeit our sample was large and included similar numbers of male and female subjects in each decade. Second, a few variables which could contribute to the variance in the ISWD were not assessed, such as pulmonary function [15-18] and peripheral muscle force [16]. However, additional equipment and expertise are necessary to collect such variables.

\section{Conclusions}

We have established a reference equation for the ISWD prediction in Japanese adults. The prediction accuracy was high $\left(\mathrm{R}^{2}=50 \%\right)$, and the equation was established using age, height, and gender which can be easily collected in clinical settings. The reference equation will be useful in evaluating the extent of exercise intolerance in Japanese samples.

\section{Conflict of interest}

The authors have no conflicts of interest. 


\section{Acknowledgments}

The authors thank the study participants, hospitals, schools, and nursing homes for their

cooperation in data collection. We also thank Dr. Sue Jenkins, of Sir Charles Gairdner Hospital, for reviewing this manuscript. This study was supported by the Japanese Respiratory Society and the Japanese Society for Respiratory Care and Rehabilitation.

\section{References}

[1] Spruit MA, Singh SJ, Garvey C, ZuWallack R, Nici L, Rochester C, et al. An official American Thoracic Society/European Respiratory Society statement: key concepts and advances in pulmonary rehabilitation. Am J Respir Crit Care Med 2013;188(8):e13-64.

[2] Butland RJ, Pang J, Gross ER, Woodcock AA, Geddes DM. Two-, six-, and 12minute walking tests in respiratory disease. Br Med J (Clin Res Ed) 1982;284(6329):1607-8.

[3] Singh SJ, Morgan MD, Scott S, Walters D, Hardman AE. Development of a shuttle walking test of disability in patients with chronic airways obstruction. Thorax 1992;47(12):1019-24.

[4] Chetta A, Pisi G, Aiello M, Tzani P, Olivieri D. The walking capacity assessment in the respiratory patient. Respiration 2009;77(4):361-7.

[5] Singh SJ, Puhan MA, Andrianopoulos V, Hernandes NA, Mitchell KE, Hill CJ, et al. An official systematic review of the European Respiratory Society/American Thoracic Society: measurement properties of field walking tests in chronic respiratory disease. Eur Respir J 2014;44(6):1447-78.

[6] Campo LA, Chilingaryan G, Berg K, Paradis B, Mazer B. Validity and reliability of the modified shuttle walk test in patients with chronic obstructive pulmonary 
disease. Arch Phys Med Rehabil 2006;87(7):918-22.

[7] Evans RA, Singh SJ, Collier R, Williams JE, Morgan MD. Pulmonary rehabilitation is successful for COPD irrespective of MRC dyspnoea grade. Respir Med 2009;103(7):1070-5.

[8] Hillerdal G, Lofdahl CG, Strom K, Skoogh BE, Jorfeldt L, Nilsson F, et al. Comparison of lung volume reduction surgery and physical training on health status and physiologic outcomes: a randomized controlled clinical trial. Chest 2005;128(5):3489-99.

[9] Murray MP, Turnbull K, Macquarrie S, Hill AT. Assessing response to treatment of exacerbations of bronchiectasis in adults. Eur Respir J 2009;33(2):312-8.

[10] Calvert LD, Singh SJ, Morgan MD, Steiner MC. Exercise induced skeletal muscle metabolic stress is reduced after pulmonary rehabilitation in COPD. Respir Med 2011;105(3):363-70.

[11] Sandland CJ, Morgan MD, Singh SJ. Detecting oxygen desaturation in patients with COPD: incremental versus endurance shuttle walking. Respir Med 2008;102(8):1148-52.

[12] Ringbaek T, Martinez G, Brondum E, Thogersen J, Morgan M, Lange P. Shuttle walking test as predictor of survival in chronic obstructive pulmonary disease patients enrolled in a rehabilitation program. J Cardiopulm Rehabil Prev 2010;30(6):409-14.

[13] Emtner MI, Arnardottir HR, Hallin R, Lindberg E, Janson C. Walking distance is a predictor of exacerbations in patients with chronic obstructive pulmonary disease. Respir Med 2007;101(5):1037-40.

[14] Tully MA, Cupples ME, Hart ND, McEneny J, McGlade KJ, Chan WS, et al. Randomised controlled trial of home-based walking programmes at and below current recommended levels of exercise in sedentary adults. J Epidemiol Community Health 2007;61(9):778-83.

[15] Dourado VZ, Vidotto MC, Guerra RL. Reference equations for the performance of healthy adults on field walking tests. J Bras Pneumol 2011;37(5):607-14.

[16] Harrison SL, Greening NJ, Houchen-Wolloff L, Bankart J, Morgan MD, Steiner $\mathrm{MC}$, et al. Age-specific normal values for the incremental shuttle walk test in a healthy British population. J Cardiopulm Rehabil Prev 2013;33(5):309-13.

[17] Jurgensen SP, Antunes LC, Tanni SE, Banov MC, Lucheta PA, Bucceroni AF, et al. The incremental shuttle walk test in older Brazilian adults. Respiration 2011;81(3):223-8.

[18] Probst VS, Hernandes NA, Teixeira DC, Felcar JM, Mesquita RB, Goncalves CG, et 
al. Reference values for the incremental shuttle walking test. Respir Med 2012;106(2):243-8.

[19] Kagawa M, Binns CB, Hills AP. Body composition and anthropometry in Japanese and Australian Caucasian males and Japanese females. Asia Pac J Clin Nutr 2007;16 Suppl 1:31-6.

[20] Nakanishi Y, Nethery V. Anthropometric comparison between Japanese and Caucasian American male university students. Appl Human Sci 1999;18(1):9-11.

[21] McKeough ZJ, Leung RW, Alison JA. Shuttle walk tests as outcome measures: Are two incremental shuttle walk tests and two endurance shuttle walk tests necessary? Am J Phys Med Rehabil 2011;90(1):35-9.

[22] Eiser N, Willsher D, Dore CJ. Reliability, repeatability and sensitivity to change of externally and self-paced walking tests in COPD patients. Respir Med 2003;97(4):407-14.

[23] Karvonen MJ, Kentala E, Mustala O. The effects of training on heart rate; a longitudinal study. Ann Med Exp Biol Fenn 1957;35(3):307-15.

[24] Astrand I. Aerobic work capacity in men and women with special reference to age. Acta Physiol Scand Suppl 1960;49(169):1-92.

[25] Bohannon RW. Population representative gait speed and its determinants. J Geriatr Phys Ther 2008;31(2):49-52.

[26] Busch Tde A, Duarte YA, Pires Nunes D, Lebrao ML, Satya Naslavsky M, dos Santos Rodrigues A, et al. Factors associated with lower gait speed among the elderly living in a developing country: a cross-sectional population-based study. BMC Geriatr 2015;15:35.

[27] Ko SU, Hausdorff JM, Ferrucci L. Age-associated differences in the gait pattern changes of older adults during fast-speed and fatigue conditions: results from the Baltimore longitudinal study of ageing. Age Ageing 2010;39(6):688-94.

[28] Tolea MI, Costa PT, Terracciano A, Griswold M, Simonsick EM, Najjar SS, et al. Sex-specific correlates of walking speed in a wide age-ranged population. J Gerontol B Psychol Sci Soc Sci 2010;65B(2):174-84.

[29] Evans WJ, Campbell WW. Sarcopenia and age-related changes in body composition and functional capacity. J Nutr 1993;123(2 Suppl):465-8.

[30] Fleg JL, Lakatta EG. Role of muscle loss in the age-associated reduction in VO2 max. J Appl Physiol (1985) 1988;65(3):1147-51.

[31] Neves CD, Lacerda AC, Lage VK, Lima LP, Fonseca SF, de Avelar NC, et al. Cardiorespiratory responses and prediction of peak oxygen uptake during the shuttle walking test in healthy sedentary adult men. PLoS One 
2015;10(2): e0117563.

[32] Hayashida I, Tanimoto Y, Takahashi Y, Kusabiraki T, Tamaki J. Correlation between muscle strength and muscle mass, and their association with walking speed, in community-dwelling elderly Japanese individuals. PLoS One 2014;9(11):e111810.

[33] Camarri B, Eastwood PR, Cecins NM, Thompson PJ, Jenkins S. Six minute walk distance in healthy subjects aged 55-75 years. Respir Med 2006;100(4):658-65.

[34] Poh H, Eastwood PR, Cecins NM, Ho KT, Jenkins SC. Six-minute walk distance in healthy Singaporean adults cannot be predicted using reference equations derived from Caucasian populations. Respirology 2006;11(2):211-6.

[35] Mikawa K, Senjyu H. Development of a field test for evaluating aerobic fitness in middle-aged adults: validity of a 15 -m incremental shuttle walk and run test. J Sports Sci Med 2011;10(4):712-7. 


\section{Appendices}

Table 1 Demographics and incremental shuttle walk test data $(\mathbf{n}=590)$.

Data are expressed as median [interquartile range $25-75 \%$ ].

BMI: body mass index. ISWD: incremental shuttle walk distance. PR: pulse rate. \%HRmax:

percentage of the predicted HRmax $(220$ - age) reached at the end of the walk test.

Completer: those who completed 12 levels of ISWT.

Table 2 Relationships between gender and anthropometric variables with incremental shuttle

walk distance.

r: Spearman correlation coefficient

Table 3 Multiple linear regression analysis with incremental shuttle walk distance as the dependent variable.

B: Unstandardized coefficients, CI: Confidence interval for B

Residual standard deviation $=145.8 \mathrm{~m}$

Reference equation: ISWD $(\mathrm{m})=-4.894-(4.107 \times$ Age $)+(131.115 \times$ Gender $)+(4.895 \times$ Height $)$ 
${ }^{a}:$ male $=1$

Fig. 1 Scatter plot of the measured ISWD and predicted ISWD from Probst et al. 
Table1 Demographics and incremental shuttle walk test data $(n=590)$.

\begin{tabular}{|c|c|c|c|c|c|}
\hline \multicolumn{6}{|c|}{ Age bands, Total n (men) } \\
\hline & 20-39 y, 249 (129) & $40-59$ y, 192 (73) & $60-79$ y, $131(30)$ & $\geq 80 \mathrm{y}, 18(5)$ & Overall, 590 (237) \\
\hline Age (years) & $28[25-33]$ & $48[44-53]$ & $69[64-74]$ & $83[81-85]$ & $44[31-60]$ \\
\hline Height $(\mathrm{cm})$ & $165[158-172]$ & $161[156-169]$ & $153[150-159]$ & $151[146-160]$ & $161[154-168]$ \\
\hline Weight (kg) & $57[50-68]$ & $57[50-66]$ & $54[49-62]$ & $54[49-58]$ & $56[50-65]$ \\
\hline BMI $\left(\mathrm{kg} / \mathrm{m}^{2}\right)$ & 20.8 [19.5-22.9] & $21.7[20.0-23.5]$ & $23.0[21.5-24.9]$ & $22.7[21.3-24.8]$ & $21.6[19.9-23.7]$ \\
\hline ISWD (m) & 750 [575-915] & $640[530-745]$ & $480[420-560]$ & $350[200-410]$ & 640 [490-793] \\
\hline Completer $(\%)$ & $49(8.3)$ & $14(2.3)$ & 0 & 0 & $63(10.7)$ \\
\hline PR before (bpm) & $78[70-86]$ & $80[72-88]$ & $73[65-82]$ & $65[63-75]$ & $77[70-86]$ \\
\hline PR end (bpm) & $151[136-164]$ & $146[125-160]$ & $120[108-141]$ & 110 [90-129] & $144[121-160]$ \\
\hline$\% \mathrm{HR} \max (\%)$ & $79[71-86]$ & 85 [74-95] & $82[71-93]$ & $79[67-94]$ & $81[71-90]$ \\
\hline $\mathrm{SpO}_{2}$ before $(\%)$ & 98 [97-99] & 98 [97-99] & 97 [97-98] & 97 [97-98] & 98 [97-99] \\
\hline $\mathrm{SpO}_{2}$ end $(\%)$ & 96 [95-98] & 96 [95-97] & 96 [94-98] & 96 [93-98] & 96 [95-98] \\
\hline Dyspnea before & $0[0-0]$ & $0[0-0]$ & $0[0-1]$ & $0[0-2]$ & $0[0-0]$ \\
\hline Dyspnea end & $4[4-5]$ & $4[3-6]$ & $4[4-5]$ & $4.5[4-5]$ & $4[4-5]$ \\
\hline Leg effort before & $0[0-0]$ & $0[0-0]$ & $0[0-1]$ & $0[0-2]$ & $0[0-0.5]$ \\
\hline Leg effort end & $5[4-7]$ & $4[3-7]$ & $4[2-5]$ & $4[2.8-5.5]$ & $5[3-7]$ \\
\hline
\end{tabular}

Data are expressed as median [ interquartile range $25-75 \%$ ].

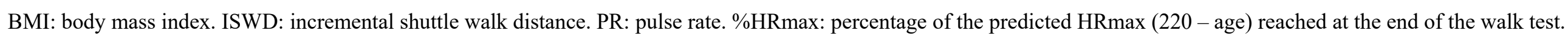

Completer: those who completed 12 levels of ISWT. 
Table 2 Relationships between gender and anthropometric variables with incremental shuttle walk distance.

\begin{tabular}{lcc}
\hline & $\mathrm{r}$ & $\mathrm{p}$-value \\
\hline Age (years) & -0.51 & $<0.001$ \\
Gender & 0.56 & $<0.001$ \\
Height $(\mathrm{cm})$ & 0.62 & $<0.001$ \\
Weight $(\mathrm{kg})$ & 0.39 & $<0.001$ \\
BMI $\left(\mathrm{kg} / \mathrm{m}^{2}\right)$ & -0.01 & 0.747 \\
\hline
\end{tabular}

r: Spearman correlation coefficient 
Table 3 Multiple linear regression analysis with incremental shuttle walk distance as the dependent variable.

\begin{tabular}{lccccc}
\hline & \multirow{2}{*}{$\mathrm{B}$} & \multicolumn{2}{c}{$95 \% \mathrm{CI}$} & Partial & p-value \\
& & Lower limit & Upper limit & correlation & \\
\hline Constant & -4.894 & -355.090 & 345.301 & & 0.978 \\
Age (years) & -4.107 & -4.859 & -3.355 & -0.355 & $<0.001$ \\
Gender $^{\text {a }}$ & 131.115 & 92.886 & 169.343 & 0.311 & $<0.001$ \\
Height $^{(\mathrm{cm})}$ & 4.895 & 2.757 & 7.034 & 0.225 & $<0.001$ \\
\hline
\end{tabular}

B: Unstandardized coefficients, CI: Confidence interval for B

Residual standard deviation $=145.8 \mathrm{~m}$

Reference equation: ISWD $(\mathrm{m})=-4.894-(4.107 \times$ Age $)+(131.115 \times$ Gender $)+(4.895 \times$ Height $)$

a: male $=1$ 


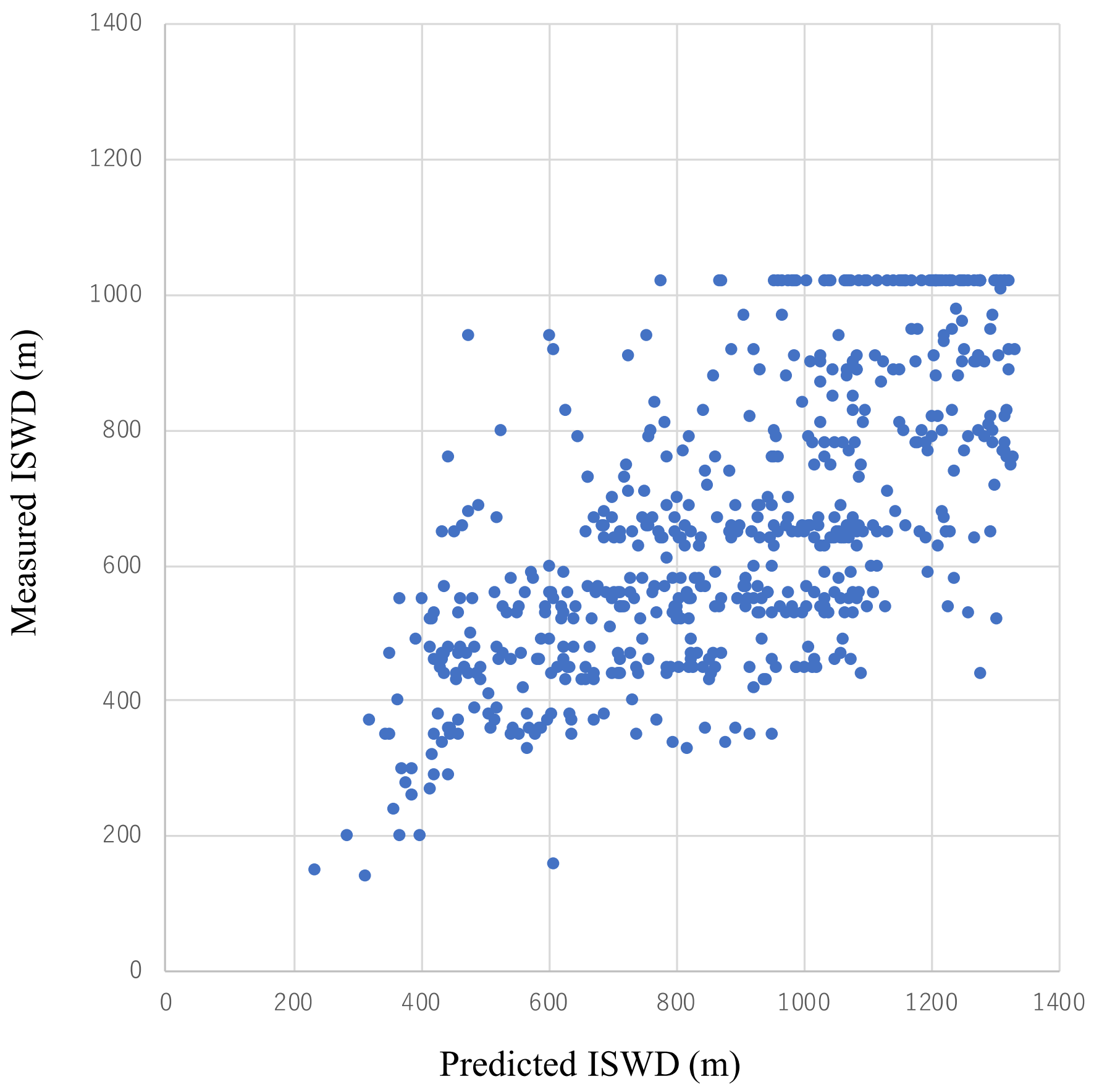

Figure 1 Scatter plot of measured ISWD and predicted ISWD from Probst et al. 